\title{
IBERIA: UN JUEGO DE ROL PARA UNA DIDÁCTICA DE LA HISTORIA ANTIGUA SIGNIFICATIVA E INNOVADORA
}

\author{
Iberia: A Role-Playing Game for an Innovative Teaching of Ancient History
}

\begin{abstract}
Víctor Sánchez Domínguez
Universidad de Sevilla. España

vsanchez1@us.es | https://orcid.org/0000-0003-1628-2914
\end{abstract}

Alfonso Álvarez-Ossorio Rivas

Universidad de Sevilla. España

alfossorio@us.es | https://orcid.org/0000-0002-9450-0369

Anthony Álvarez Melero

Universidad de Sevilla. España

aalvamel@us.es | https://orcid.org/0000-0002-3788-2567

Fecha de recepción: 13/10/2021

Fecha de aceptación: 15/02/2022

Acceso anticipado 08/03/2022

Resumen: Esta aportación refleja los resultados de la investigación realizada durante el curso 2018/19 en relación con la introducción juegos de rol en la didáctica de la Historia Antigua en Educación superior.

Ante la problemática detectada en diferentes titulaciones con respecto al nuevo alumnado y adaptándonos a las exigencias del aprendizaje competencial, se ha diseñado como alternativa metodológica un juego de rol de contenido histórico, Iberia. Este juego se ha aplicado en diferentes grupos y se ha evaluado su impacto en el alumnado como recurso didáctico a la hora de enseñar Historia Antigua en general y de la península ibérica en particular.

Durante el curso 2018/19 ha realizado una prueba piloto del juego diseñado con nueve grupos de entre 30 y 60 alumnos y se han tomado tres de ellos como muestra, siendo encuestados sobre la actividad por medio de un cuestionario elaborado por el equipo docente. 
Los resultados obtenidos de la prueba piloto por medio de la observación directa y la elaboración y uso de un cuestionario plantean una clara aceptación del recurso para las clases prácticas, acercando al alumno a temas concretos como son los procesos económicos y sociales propios de la Historia Antigua de la península ibérica, y ha provocado una mejoría en la comprensión de estos por parte del alumnado.

Palabras clave: Historia Antigua; juego de rol; educación superior; gamificación; innovación educativa.

Abstract: This contribution reflects the results of the research carried out during the 2018/19 academic year in relation to the introduction of role-playing games in the didactics of Ancient History in Higher Education.

Given the problems detected in different degrees with respect to new students and adapting to the demands of competency learning, a role-playing game with historical content, Iberia, has been designed as a methodological alternative. This game has been applied in different groups and its impact on students has been evaluated as a didactic resource when teaching Ancient History in general and the Iberian Peninsula in particular.

During the 2018/19 academic year, a pilot test of the designed game has been carried out with nine groups of between 30 and 60 students and three of them have been taken as a sample, being surveyed about the activity through a questionnaire prepared by the teaching team.

The results obtained from the pilot test through direct observation and the elaboration and use of a questionnaire raise a clear acceptance of the resource for practical classes, bringing the student closer to specific topics such as the economic and social processes of Ancient History of the Iberian Peninsula, and has led to an improvement in the understanding of these by students.

Keywords: Ancient history; role playing; Higher Education; gamification; educational innovations.

Sumario: 1. Introducción; 2. Planteamientos teóricos, nuevas metodologías docentes en educación superior; 2.1. El reto del EEES y la didáctica de la Historia Antigua; 2.2. EI LARP definición y potencial didáctico; 3. Metodología; 3.1. Objetivos; 3.2. Contexto y muestra; 3.3. Diseño del recurso; 3.4. Diseño del cuestionario; 4 .Resultados y discusión; 5 . Reflexiones finales; 6 . Referencias bibliográficas.

\section{INTRODUCCIÓN}

Esta aportación se centra en el análisis del potencial de los juegos de rol, concretamente de los juegos de rol en vivo o LARP (Live Action Role-Playing) como recurso didáctico para mejorar el proceso de enseñanza-aprendizaje de la Historia Antigua para los alumnos de diferentes titulaciones. En ella presentamos el diseño y resultado de una parte del proyecto de innovación docente De la teoría a la práctica: gamificación y juegos de rol en las clases de historia, concretamente la 
centrada en el objetivo de introducir y utilizar el LARP como recurso didáctico, así como investigar su impacto en el alumnado ${ }^{1}$. Esta investigación, que busca evaluar el impacto de la innovación docente, se encuadra dentro de un proyecto más amplio centrado en fomentar el uso de las nuevas metodologías activas en la educación superior y tiene como fin último mejorar el proceso de enseñanzaaprendizaje en la didáctica de las Ciencias Sociales en general y de la Historia Antigua de manera concreta.

Así pues, esta investigación entronca con una serie de estudios en los que los autores vienen participando en los últimos años dedicados al análisis de las dificultades en la didáctica de los procesos históricos y más concretamente de la didáctica de la Historia Antigua en la educación superior y los nuevos retos que de ellos surgen (Sánchez Domínguez, Álvarez-Ossorio, Alarcón, y Lozano, 2018 y Sánchez Domínguez, Álvarez-Ossorio y Lozano, 2019). A través de investigaciones realizadas en diferentes proyectos de innovación docente hemos constatado la necesidad de modificar tanto las dinámicas de clase como los recursos empleados en una búsqueda por adaptarnos al nuevo alumnado que llega a la universidad.

Esta aportación recoge en primer lugar una disertación sobre los retos derivados del nuevo marco Europeo de Educación Superior (EEES) y cómo ha afectado a los procesos de enseñanza y aprendizaje de los nuevos títulos, centrándonos muy especialmente en la problemática de la didáctica de la Historia y concretamente de la Historia Antigua, un área de conocimiento que se aleja o aproxima al alumnado de manera desigual debido a su preespecialidad en diferentes canales de comunicación actual. Es por esta razón que buscamos su adaptación a metodologías más próximas al alumnado, que permitan un mejor desarrollo competencial.

Tras esta disertación se presentará el concepto del LARP y Edu-LARP como metodología activa, su vinculación con la gamificación y el aprendizaje basado en juegos, para plantearlo como alternativa metodológica que complemente los modelos expositivos tradicionales, aprovechando las nuevas secuenciaciones en los títulos, así como la obligatoriedad de sistemas de prácticas.

Una vez expuesta la teoría que sustenta este proyecto, se pasará a presentar el diseño y aplicación del juego Iberia en una prueba piloto con el alumnado de la asignatura Fundamentos de Historia, añadiendo además el diseño de un cuestionario a modo de instrumento de investigación educativa que recabe los datos de las percepciones del alumnado.

${ }^{1}$ La financiación de los proyectos ha corrido a cargo del II y III Plan Propio de Docencia de la Universidad de Sevilla. 


\section{PLANTEAMIENTOS TEÓRICOS, NUEVAS METOdOLOGÍAS DOCENTES EN EDUCACIÓN SUPERIOR}

\subsection{El reto del EEES y la didáctica de la Historia Antigua}

Los propios planteamientos del sistema universitario y su adaptación al nuevo marco Europeo de Educación Superior (EEES) apuntaban ya, en el momento de implantación de los nuevos planes de estudios, hacia la necesidad de una reconversión del proceso de enseñanza aprendizaje centrándose más en el alumno y en su desarrollo competencial. Diferentes autores como Alfaro Rocher (2005), Benito y Cruz (2005), De Miguel (2006), Cárdenas (2015) y Vázquez García (2015) recalcaron en su momento la necesidad que existía de desarrollar este proceso de cambio, y muchos aprovecharon para hablar de la necesidad de incorporar nuevas metodologías docentes en las que el papel del profesor fuera modificado. Recientemente Jiménez et al. (2020) expresaban cuán importante eran estas metodologías y junto a autores como Calvo y Mingorance (2009) o Palomares (2011) apuestan por su aplicación recalcando el papel que desempeñaban en el desarrollo de las competencias del alumnado. Este planteamiento de Jiménez et al. (2020) retoma las líneas y el análisis de De Miguel (2005) sobre los métodos de enseñanza en la educación superior y su organización, centrándose en el grado de participación del alumnado y la necesidad de incorporar el concepto de enseñanza activa a la docencia universitaria, uno de los problemas que queremos abordar.

Para nosotros es necesario que el alumno tome la guía de su propio proceso de aprendizaje, una visión que comparte algunos puntos con las corrientes que abogan por dar protagonismo a los estudiantes y a la metodología frente al profesor y los contenidos en la educación superior (de Alba-Fernández y Porlán, 2017; de Alba-Fernández et al., 2020). Si bien desde nuestra experiencia vemos que los contenidos son muy necesarios y se deben trabajar, entendemos que es necesario incorporar nuevas estrategias que faciliten su proceso de aprendizaje. Queremos apostar por esta nueva realidad potenciando el desarrollo y la incorporación de nuevas metodologías de enseñanza mucho más activas.

Esta tendencia basada en la incorporación de metodologías activas no es ajena a la didáctica de la Historia, ni a la didáctica de la Historia Antigua en educación superior. Tampoco es exclusiva de nuestros proyectos como se aprecia en diferentes ejemplos como el de Cardete centrado en el aprendizaje cooperativo y el uso de plataformas digitales (Cardete, 2011), el de San Bernardino basado en el aprendizaje por resolución de problemas (San Bernardino, 2019) o experiencias como las de Alarcón (2016) basadas en el aprendizaje crítico, estas dos últimas potenciadas por el programa FIDOP que la Universidad de Sevilla utiliza para crear ciclos de mejora en la docencia Universitaria involucrando a profesorado de diferentes departamentos y áreas en el desarrollo de proyectos de innovación docente de manera periódica. 
Es en este escenario de cambio y adaptación en el que se encuadran nuestros proyectos de innovación para la docencia de la asignatura sobre la que hemos trabajado, "Fundamentos de Historia, Historia de España». Esta asignatura se incorporó al plan de estudios en los grados de educción en diferentes universidades dentro de las titulaciones de Grado y Doble Grado en Educación primaria. Desde su origen se han detectado diferentes problemáticas derivadas del propio alumnado en cuanto a su nivel de conocimientos iniciales, así como sus aspiraciones y motivación; de los contenidos, en cuanto a su grado de complejidad, vinculación con conocimientos previos y extensión; así como en su didáctica, en relación a la perduración de sistemas docentes expositivos, los recursos utilizados y el impacto que producen en los alumnos e incluso con respecto a los niveles de exigencia (Sánchez Domínguez, Álvarez-Ossorio y Lozano, 2019, pp. 348-351). Estos problemas que detectamos se plantean como una realidad extendida, así Alarcón habla del desinterés del alumnado frente a la historia (2016, pp. 886-887), Espinel Souares (2007) plantea nuestras mismas tesis sobre el distanciamiento del alumno y la necesidad de modificar la enseñanza de la Historia en general y la Historia Antigua en particular para conseguir un aprendizaje significativo. Es este autor quien demuestra que es posible conseguir este tipo de aprendizaje, pues, tras un estudio cualitativo, aprecia en sus conclusiones que la Historia Antigua no está tan alejada de nuestro alumnado universitario (Espinel Souares, 2007, pp. 152-153). Es más, el estudio de Ruiz López (2020), realizado en una muestra muy similar a la nuestra, apoya el planteamiento basado en que el alumno tiene una visión propia de la Historia Antigua, lejana a los contenidos que explicamos y extraída principalmente de la cultura popular por medio del cine, la televisión y el contacto con el patrimonio local. En nuestra opinión, esto hace que tengan una visión sesgada de los contenidos y unas expectativas diferentes a lo que encuentran en el aula, pues, como recuerda Cardete (2011, pp. 143-144), mientras que la historiografía de nuestra área de conocimiento ha sufrido fuertes cambios en las últimas décadas, nuestra metodología docente sigue basándose en el aprendizaje memorístico.

Debido a todo esto, y dentro de este nuevo escenario y tras analizar contenidos y recursos, se ha procedido a realizar diferentes proyectos de innovación en los que, centrándonos en el alumno, le hemos proporcionado nuevos materiales (Sánchez Domínguez, Álvarez-Ossorio, Alarcón, y Lozano, 2018), nuevos recursos y hemos introducido nuevas metodologías docentes que buscan facilitar la didáctica de la Historia Antigua (Álvarez-Melero, Álvarez-Ossorio, Cidoncha, y Sánchez Domínguez, 2018).

EI LARP se plantearía por tanto como uno de estos nuevos recursos didácticos, y se enmarcaría en una metodología docente de Aprendizaje Basado en Juegos y dentro de la definición de Serious Game, entendiendo que el LARP no es un juego de roles como un psico-drama o una improvisación común, sino que viene asociado a un conjunto de reglas que permiten la interacción en la vida real de personajes ficticios a partir de la interpretación de los participantes de esta actividad. Pero antes de continuar sería necesario aclarar qué es el LARP y su importancia como recurso educativo. 


\subsection{EI LARP definición y potencial didáctico}

Sara Lyne Bowman (2010) definió el LARP como:

a form of game play in which participants physically embody characters within a fictional scenario for extended periods of time. Designers can set larps in any time, place, or genre. Characters range from strongly similar to the player's primary identity to completely distinct (2010, citado en Bowman y Standiford, 2015, p. 4).

Así, autores como Peterson y Vanek hablan de diferentes niveles de LARP y lo asocian al psico-drama desarrollado por Moreno a principios de siglo xx o a los ejercicios de improvisación propios del teatro (Peterson y Vanek, 2016). Torstein argumenta que este juego se encontraría dentro de las categorías de las paidia definidos por Caillois con un fuerte componente de mímica, un alto grado de libertad y pocas reglas (Torstein, 2005). No obstante, y aunque guarden relación, como se aprecia en los diferentes trabajos que abordan los orígenes del LARP desde una perspectiva amplia (Tychsen et al., 2006, pp. 256-285; Mochocki, 2013), existe un consenso por parte de los autores en vincular el LARP a los juegos de rol, de donde surgirían y tomarían sus reglas. Como plantea Maragliano, la diferencia entre el LARP y el juego de rol de tablero radicaría en que mientras en este último el jugador interpreta a un caballero que busca un tesoro a través de la imaginación o un tablero, pongamos ese ejemplo, en el LARP, el jugador intenta ir disfrazado de caballero y busca el tesoro en un bosque o espacio real (Maragliano, 2019, párr. 3).

Autores como Tychsen, Hitchens y Brolund plantean que el LARP es una variante de los juegos de rol y exponen cómo comparte todos los elementos de los juegos de tablero con ligeros cambios para adaptarlos a un escenario de juego real (Tychesen et al., 2006, pp. 254-255). Las propias siglas LARP, extraídas de Live Action Role-Playing, se traducen al castellano como rol en vivo, una modalidad dentro de los juegos de rol en la que los participantes interpretan a sus personajes en un entorno real, entendiendo real por físico y no completamente imaginario. Así, la definición de Amézquita-Castañeda \& Moreno-Ramos (2001) sobre qué es un juego de rol podría aplicarse al concepto de LARP partiendo de la distinción antes hecha, encontrándonos con que es:

[...] un proceso interactivo en un marco de cuantificación cuya finalidad es la narración. El objetivo de un juego de rol es contar una historia en la que los personajes adoptados por los jugadores son los héroes. El juego es interactivo porque las decisiones del jugador conducen la historia y el final varía dependiendo de lo que harán los jugadores. (p. 34). 
Como afirma Rangel Jiménez, los juegos de rol (tanto de mesa como LARP) tratan de construir «una narrativa en y desde la mente de los jugadores» $(2015, \mathrm{p}$. 3390). Mackay (2001) los define como un sistema de creación de historias episódicas y participativas que, incorporando reglas, permite a un grupo de jugadores y un director de juego determinar cómo se resuelven las interacciones entre los personajes que interpretan. Craig y Eladhari (2005) llegan a plantear una gran familia de juegos de rol y hablan de juegos de rol de mesa o tablero y juegos de rol en vivo, en los que los jugadores se disfrazan e interpretan sus personajes en la vida real, juegos de rol en plataforma virtual del tipo RPG, multijugadores en RPG y juegos de rol trans-reales y Roda (2015, pp. 193-195), tras unir los RPG y los MMORPG (Massively Multiplayer Online Role-Playing Game), habla del juego de rol por escrito.

Estas características de juego reglado, juego inmersivo, interpretativo, etc., hacen a autores como Antonio Roda decir que «el juego de rol se muestra como una herramienta que sobre todo permite desarrollar las capacidades de lectura, interacción social, escritura e imaginación», una herramienta para crear historias (2010, p. 200).

Este planteamiento no ha pasado desapercibido para los profesores de Historia, y así, el uso de los juegos de rol tradicionales como estrategias de aprendizaje ya había sido trabajado por compañeros como Carbó y Pérez Miranda (2010) y desde nuestro equipo se está estudiando la vinculación de estos juegos con metodologías de Gamificación y Aprendizaje Basado en Juegos (Sánchez Domínguez, 2018). Sin embargo, las limitaciones de los RPG de mesa tradicional e incluso de los digitales nos empujaron claramente a abordar la posibilidad de implementar el LARP para la didáctica de nuestra asignatura de Historia. Un salto que entronca con toda una corriente de pensamiento que habla del Edu-LARP (Bowman, 2010; Mochocki, 2013; Maragliano, 2019 o Lacanienta, 2020).

Así, a la concepción de esta estrategia, su carácter lúdico y su vinculación con el concepto de Juego, tan fundamental en la educación para autores como Vygotsky (1982), Dewey (1997) o Huizinga (2000), se le une la acción y participación activa que fomenta conceptos como el aprendizaje significativo y sienta las bases para un claro constructivismo social en el que los participantes van adquiriendo unas ideas previas de la realidad interpretada que son incrementadas con la interacción y estimulación provocada por otros individuos.

Dentro de la dinámica de mejorar la motivación y el aprendizaje de nuestra materia, entendimos que la incorporación de esta metodología docente utilizada en diferentes áreas como la psicología y las ciencias políticas sería especialmente útil. No debemos olvidar que, desde esta perspectiva, el juego de roles, una de las variantes anteriores de este concepto de LARP, ha sido útil como elemento de desarrollo personal, psicológico y pedagógico.

Este planteamiento tan globalizador del LARP proviene de su propio origen como evolución de los juegos de tablero o de rol en papel, como ya han estudia- 
do diferentes autores (Tychsen et al., 2006; Mochocki, 2013; Sánchez Domínguez, 2019). Así, se podría decir que el LARP buscó dinamizar y amplificar las experiencias obtenidas en estos juegos, que tenían limitaciones en cuanto al número de participantes, para crear un nuevo sistema de juego donde la dependencia de elementos narrativos personalizada por la figura del director de juego quedaba reducida a su mínima expresión al fortalecer el sistema de reglas y dotar de más autonomía en la interpretación a los participantes.

El alumnado, como ya hemos explicado, se encuentra perdido y desmotivado en el estudio de unos contenidos de Historia Antigua que, primero, distan de su formación por la separación en el currículum de las diferentes etapas educativas en la que se abordan; segundo, debido a la poca aplicabilidad que les encuentran, ya que aún desconocen la profundidad de los conocimientos profesionales que puede requerir la didáctica de la historia en la etapa de primaria, y en tercer lugar la ratio de alumnos por clase es elevada teniendo en los grupos reducidos una constante de en torno a 25-30 alumnos.

Por tanto, queríamos una metodología de inmersión que fomentara el aprendizaje significativo en diferentes ámbitos y que se pudiera utilizar con grandes grupos, para lo cual entendimos que un LARP adaptado a nuestros contenidos con un sistema de reglas dinámico que potenciara la participación era lo más idóneo.

El principal reto que supone el diseño de un LARP deriva del propio sistema de reglas, el cual debe ser por un lado simple para que sea memorizado por los participantes, pero a la vez debe cubrir las diferentes posibilidades que surjan de la interacción de los mismos. Además, los participantes en LARP suelen tener una experiencia previa con sistemas similares de RPG, están acostumbrados a la interpretación y a la imaginación de contextos ficticios, elementos fundamentales de este tipo de experiencias.

\section{METOdOLOGÍA}

La presente investigación se ha realizado por medio del diseño y aplicación de una propuesta de innovación basada en el LARP como recurso educativo. Tras presentar la problemática del alumnado y el concepto de LARP como recurso, se planteó diseñar y probar la propuesta que presentamos en diferentes grupos de la asignatura de "Fundamentos de Historia: Historia de España» en los Grados y Dobles Grados de Educación Primaria analizando sus reacciones en las diferentes sesiones y seleccionando una muestra a de la que se recabó información por medio de un cuestionario al término de la actividad. Esta investigación permitiría en un futuro mejorar y extender el uso del recurso diseñado a otras asignaturas que compartieran los contenidos de Historia Antigua e Historia Antigua de la península ibérica.

Así, para la realización de la investigación se llevaron a cabo diferentes fases: 
1. En primer lugar, en el transcurso de los primeros meses curso 2018-19 se recabó información sobre el LARP, sus ventajas e inconvenientes y se contactó con una empresa especializada en el diseño de eventos de LARP con la que trabajar conjuntamente en el diseño del recurso.

2. En segundo lugar, tras un proceso de revisión en los meses de diciembre y enero, se prepararon los materiales y se diseñaron los cuestionarios para llevar a cabo el experimento de aplicación.

3. En tercer lugar, durante los meses de febrero y marzo se aplicó el recurso en diferentes grupos, tomando nota de los comportamientos de los alumnos en cada una de las tres sesiones de las que se componía el recurso.

4. En cuarto lugar se pasaron los cuestionarios por medio de la plataforma Google Forms y se analizaron.

\subsection{Objetivos}

De esta manera, los objetivos que nos hemos marcado con este proyecto tienen una vertiente múltiple incluyendo elementos investigativos y de innovación, orientados tanto hacia el alumnado que cursa esta y otras asignaturas como al grupo de docentes que imparten la asignatura en sus diferentes grupos y su posibilidad de extrapolar la metodología a otros grupos docentes y asignaturas similares.

En relación con los alumnos los objetivos principales que se marcaba el proyecto de innovación docente eran:

- Desarrollar sus capacidades de análisis sobre los contenidos, así como ser capaces de establecer un debate sobre estos.

Para ello era necesario que el alumno identificara los puntos claves, las informaciones más relevantes para la enseñanza y, por tanto, la herramienta debía presentar de manera clara contenidos esenciales de la materia.

- Generar una metodología docente que ayudara a superar el marco tradicional que presentan las asignaturas de Historia Antigua.

Aunque este objetivo se puede dirigir tanto a los docentes como a los discentes debemos recordar que las percepciones del alumnado apuntan a que las clases son excesivamente teóricas, presentan sobreabundancia de textos y apenas tienen en cuenta el elemento práctico de la docencia, a pesar de que los planes de estudio de los Grados determinan que prácticamente un tercio de la docencia debe ser de carácter práctico. 
- Fomentar la evaluación continua por los propios alumnos con la vista a la exposición de los resultados de su trabajo mediante las herramientas que se les facilita.

Una constante en todos los proyectos de este equipo ha sido el modificar el sistema de evaluación buscando un mayor protagonismo del alumnado por medio de la actividad diseñada con un sistema de autoevaluación que entronque con los objetivos antes expuestos. Debemos aclarar cómo estos objetivos metodológicos se centran en el desarrollo de un nuevo método participativo de prácticas en el ámbito del conocimiento del Mundo Antiguo.

- Permitir a los alumnos ejercitar habilidades fundamentales para el ulterior desarrollo de su vida académica y profesional generando siempre una atmósfera de pluralidad y tolerancia, así como fomentar el desarrollo de la capacidad crítica entre el alumnado.

Todo esto se enmarca en los objetivos que han venido reiterándose en todos los proyectos y se centra en la necesidad de dar sentido a los contenidos propios de Historia y vincularlos con el desarrollo profesional de una manera no memorística sino activa, que pueda ser utilizada en entornos reales al desarrollar el espíritu crítico.

- Introducir una mecánica alternativa para la enseñanza de la Historia Antigua, basada en la gamificación y el juego de rol, y probarla mediante la combinación de nuevas y «tradicionales» formas de evaluación.

Este último objetivo de nuevo tiene una doble vertiente formativa tanto para los discentes que cursan la asignatura y que les permite aprender nuevas metodologías docentes que fomenten el aprendizaje significativo de los contenidos propios de la Historia Antigua y a la vez para los docentes, quienes, a través de este proyecto, realizan un proceso de autoformación didáctica al experimentar la enseñanza por medio de nuevas metodologías docentes.

\subsection{Contexto y muestra}

Como se aprecia en los objetivos, estos no se circunscribían en un primer momento solo a la asignatura de Fundamentos, sino que englobaban un conjunto más amplio de asignaturas dependientes del Dpto. de Historia Antigua. Sin embargo, el grupo de docentes implicados en los proyectos y las características de esta asignatura la hacían especialmente idónea para nuestra propuesta.

En primer lugar, nos encontramos que la totalidad del profesorado que impartiría los contenidos propios de Historia Antigua dentro de esta asignatura esta- 
ban inscritos en el proyecto de innovación docente, siendo el núcleo un grupo de docentes que había impartido la asignatura desde su implantación y que ya tenía experiencia en proyectos de innovación educativa en el seno de esta misma asignatura.

En segundo lugar, nos enfrentamos a un alumnado con características muy especiales:

Una de ellas fue la edad media, de veinte a veintidós años; hecho que implicaba que el alumnado estuviera cursando una asignatura de segundo curso y presentara al menos un año ya de experiencia en la universidad. Esta experiencia previa, no existente en otras asignaturas del proyecto vinculadas a los primeros años del grado en Historia, llevaba aparejada una experiencia con diferentes metodologías docentes en educación superior.

Vinculada con esta característica, nos encontramos con que los alumnos que compondrían la muestra tendrían además una formación didáctica previa carente en el alumnado de los grados de Historia, Geografía e Historia o Arqueología. Su paso por una facultad de Ciencias de la Educación en la que se abordan a diario los problemas derivados del proceso de enseñanza y aprendizaje haría que la muestra pudiera afrontar las cuestiones didácticas de nuestra investigación de manera directa y sin necesidad de adaptaciones.

En tercer lugar, la muestra era numerosa y tenía cierta variabilidad, ya que se incorporaron los grupos del centro principal, así como otro perteneciente al centro adscrito. En el centro principal teníamos una estimación de 9 grupos de entre 40 y 70 alumnos y entre 45-55 en el centro adscrito. Esto nos permitió poder tener diferentes grupos, generando grupos de control y permitiéndonos tener acceso a un elevado número de discentes a los que encuestar sobre la experiencia.

\subsection{Diseño del recurso}

Como ya se ha comentado con anterioridad para el diseño del recurso nos pusimos en contacto con una empresa profesional de diseños de actividades de tipo LARP (Alter Ego) que diseña cenas larpificadas en las que mezcla concepto de LARP, juegos y gastronomía para grupos variables de entre 10 a 50 personas. Concretamente al inicio del proyecto estaba en el proceso de testeo de cenas de empresas para alto número de comensales, lo que aunó nuestros intereses al buscar un recurso que sirviera para grupos de desdoble de prácticas de entre 20 y 50 personas.

La participación de su director y diseñador principal Marcos López nos abrió un nuevo abanico de posibilidades ya que rápidamente entendió las particularidades del alumnado y nuestros objetivos didácticos, y así pudo implementar un sistema donde además de regular las interacciones interpretativas de los participantes, incorporaba un juego de gestión de recursos y de acciones interpersona- 
les que potenciaban la jugabilidad, a la vez que introducía conceptos económicos, sociales y políticos que eran difíciles de interpretar sin unos conceptos previos pero que, con el nuevo sistema, se aprendían a usar de manera sencilla y dinámica (Figura 1).

Así pues, organizamos grupos de trabajo entre los profesores que impartían la asignatura y con el objetivo de diseñar los materiales de contenido histórico que componían la narrativa principal del juego, así como personajes de carácter histórico o pseudo-histórico. Para el diseño de personajes se intentó que tuvieran un alto grado de veracidad y un trasfondo histórico que estuviera vinculado al temario que planteábamos gamificar, para este proyecto, concretamente la llegada de Roma a la península ibérica. Planteamos la creación de tres grandes escenas o actos que abordaran tanto la realidad indígena, el elemento púnico y helenístico, así como el romano. De esta manera, se crearon tres eventos multitudinarios con sus propias narrativas y se organizaron en torno a tres sesiones de clase: una reunión entre embajadores de los diferentes pueblos para tratar el tratado del Ebro del 226 a. C., una asamblea de pueblos íberos para debatir sobre si ayudar o no a Sagunto, sitiada por Aníbal Barca (219 a. C.) y una asamblea indígena previa a la revuelta del 197 a. C.

En la imagen que adjuntamos más abajo se puede observar cómo se crearon diferentes facciones para los jugadores: romanos, cartagineses, griegos, íberos, turdetanos, celtíberos y celtas, con características y objetivos propios. Dentro de las mismas diseñamos un total de diez personajes por facción con sus historias e interrelaciones personales entre miembros de la misma facción y con los de otras a fin de potenciar la interacción entre participantes. En total, el diseño final del juego permitía su uso con grupos de entre 21 y 70 jugadores en que todos pudieran relacionarse, fueran de la facción que fueran (Figura 2).

Además, el diseñador, Marcos López, generó todo un sistema de gestión de recursos por medio de intercambio de cartas entre personajes que implementó elementos de gamificación al diseño del LARP. Así, a diferencia de un juego de rol convencional (en vivo o en mesa), en este juego había un sistema de puntos vinculado a conseguir cartas, a ejecutar acciones especiales por medio de cartas que repartían los profesores, infiltrados en el juego al hacer uso de las identidades de los personajes que hubieran quedado vacantes (Figura 3). Se generaron tablas de puntuación a modos de rankings y los participantes podían, hasta cierta medida, modificar los hechos históricos (Figura 2). Para que los participantes se pudieran preparar para cada sesión se les facilitaron textos explicativos redactados por el equipo docente, así como resúmenes de la situación geopolítica de cada momento. Además, se diseñaron parlamentos discursivos para que ciertos personajes los recitaran en ciertos momentos de cada acto a fin de mostrar el desarrollo de acontecimientos históricos y vincularlos aún más con la actividad. 


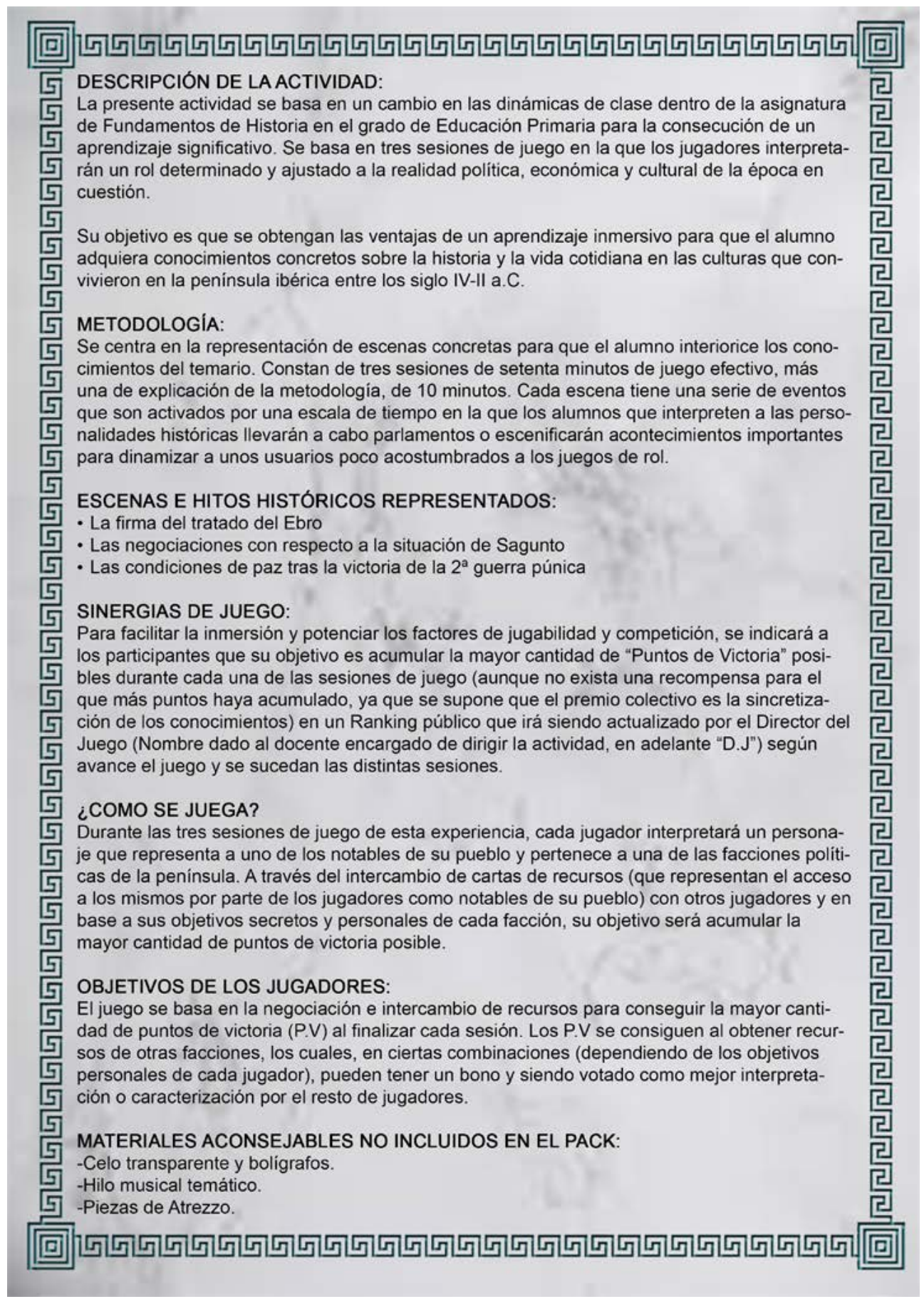

Figura 1. Instrucciones de Juego Iberia 


\section{HOJA DE CONTROL ACTO I}

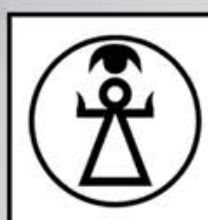

01: ANIBAL

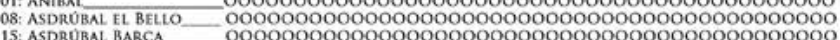

0000000000000000000000000000000000000000000

15: ASDRO BA

29: HIMILCON

36: MAGON

50: ADERBAL

57: HANNON

64: BADERTANIT

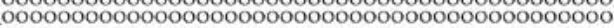
00000000000000000000000000000000000000000000

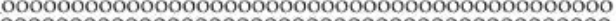
0000000000000000000000000000000000000000000 (1) 年

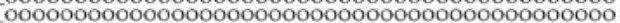

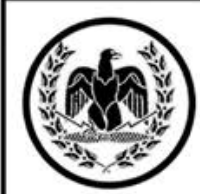

02: MARCo Catón

Oo000000000000000000000000000000000000000000 09: MARCO PAULO 16: CORNELIO ESCIPION 23: LENTULO MARCIAT 30: MARCO AUFIDIO 44: GAIO LASCENA 51: EUSEBIO MARCO 58: LUCIO AURELI —OOOOOOOOOOOOOOOOOOOOOOOOOOOOOOOOOOOOOOOOOOOO o0000000000000000000000000000000000000000000 00000000000000000000000000000000000000000000 00000000000000000000000000000000000000000000

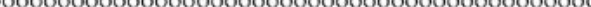
10000000000000000000000000000000000000000000 (2000000000000000000000000000000000000000000

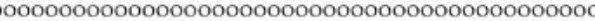

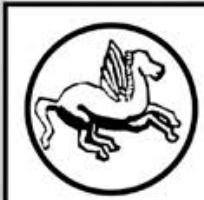

07: ARTEMIDORO

14: POLICRATE

21: EUGENIO

35: FILDEMO

42. ANDROCITES

49. MENODORO

56: ASPASIA

63: ANDRISCO

70: FILOCRATES

0ooooooooooooooooooooooooooooooooooooooooooo 00000000000000000000000000000000000000000000 000000000000000000000000000000000000000 00000000000000000000000000000000000000000000  00000000000000000000000000000000000000000000 oooopoopoopoopoopoopoopo00000000000000000000

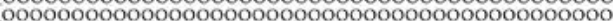

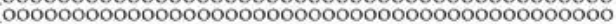

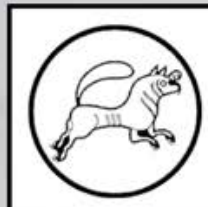

03: FEROCIO

10: CULCHAS

17: LUXINO

31: KALAITO

38: ATENNES

45: BILESETON

52: DELENINA

66: NEITIN

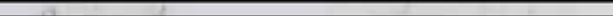

oooooooooooooooooooooooooooooooooooooooooooo

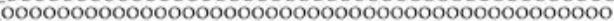
00000000000000000000000000000000000000000000 000000000000000000000000000000000000000000 00000000000000000000000000000000000000000000 00000000000000000000000000000000000000000000 00000000000000000000000000000000000000000000

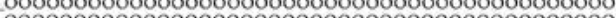

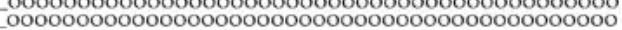

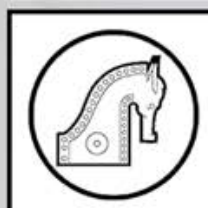

04: MUCRO

11: Orusor

18: ALCON

25: ALORCO

32: HIMILCE

39: NISUNIN
46: BASITIR

53: ALETAUNIN

53: ALETAUTI

67: MANDONIO
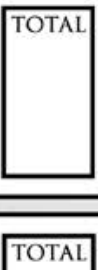

05: ALDA

12: ALUCIO

19: MELMON

26: AUSA

33: ABLON

40: TURIBA

S4: STENA

61: SICOUNIN

68: CARMERON

00000000000000000000000000000000000000000000

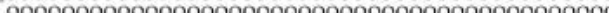

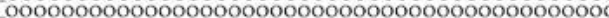
00000000000000000000000000000000000000000000 (1)  (1) 00000000000000000000000000000000000000000000

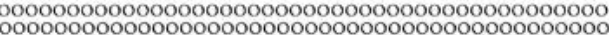

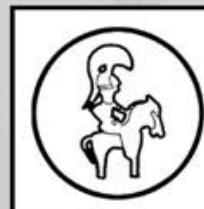

00000000000000000000000000000000000000000000

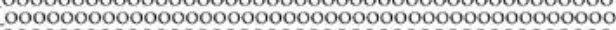
00000000000000000000000000000000000000000000 00000000000000000000000000000000000000000000

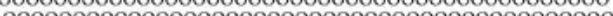
(1) 00000000000000000000000000000000000000000000 00000000000000000000000000000000000000000000 00000000000000000000000000000000000000000000

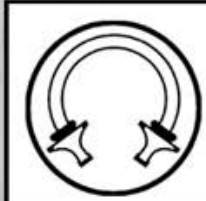

06: TILECO

13: LUBBOS

20: AMIA

27: EBURO

34: NAVIA

41: THURRO

48: AMBOLO

55: ALBURA

62: AMBATO

69: SARGA

00000000000000000000000000000000000000000000 oooopoopoopo00000000000000000000000000000000 0000000000000000000000000000000000000000000 00000000000000000000000000000000000000000000 00000000000000000000000000000000000000000000 00000000000000000000000000000000000000000000 00000000000000000000000000000000000000000000 o0000000000000000000000000000000000000000000 oooooooooo

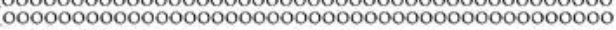
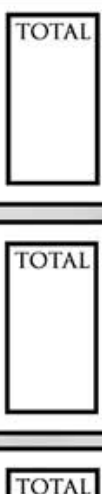

TOTAL

Figura 2. Hoja de control de personajes del Juego Iberia 


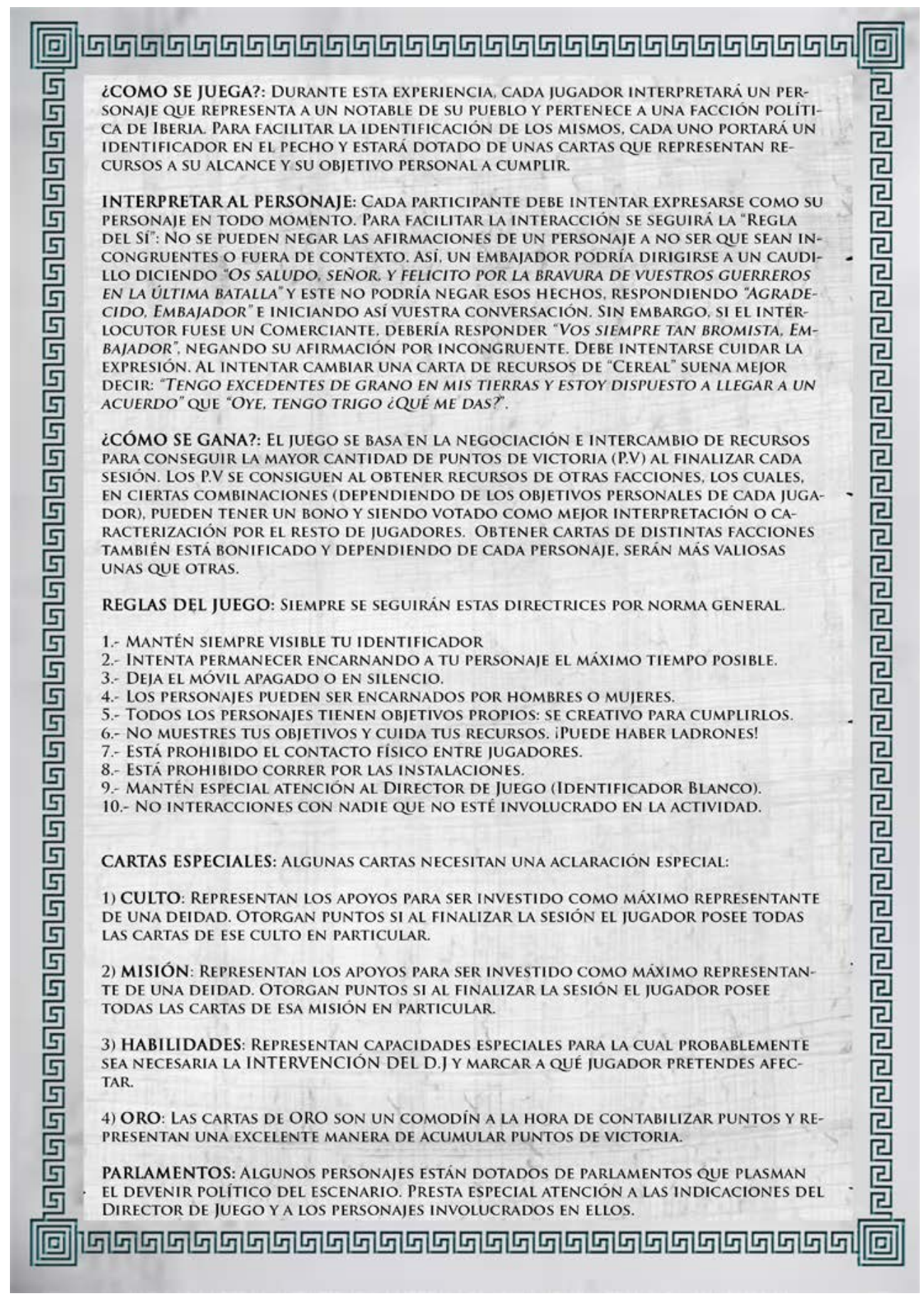

Figura 3. Instrucciones II. del Juego Iberia 


\subsection{Diseño del cuestionario}

Para ver las percepciones del alumnado frente al desarrollo de la actividad el profesorado de la asignatura diseñó un cuestionario con 23 ítems en los que se mezclaron diferentes tipos de preguntas, siendo cinco preguntas de dicotómicas, tres de opción múltiple, cinco preguntas organizadas en escalas de Likert de 5 e incorporando nueve preguntas abiertas en las que el alumno pudiera expresar con sus palabras sus percepciones.

Estos ítems se les presentaron en un formulario de Google aleatorizado, aunque se distinguen como se puede observar en la tabla, diferentes categorías (Tabla 1):

Tabla 1

\begin{tabular}{|c|c|c|}
\hline Categoría & Ítem & $\begin{array}{l}\text { Tipo de } \\
\text { pregunta }\end{array}$ \\
\hline Control & $\begin{array}{l}\text { ¿Has participado en la actividad Iberia? } \\
\text { ¿En qué centro has realizado la actividad? }\end{array}$ & $\begin{array}{l}\text { Dicotómica } \\
\text { Múltiple }\end{array}$ \\
\hline Exp. Previa & $\begin{array}{l}\text { ¿Has participado alguna vez en una actividad de LARP (rol en } \\
\text { vivo) o role play masiva? } \\
\text { ¿Cómo valorarías tu conocimiento sobre la Hispania prerromana } \\
\text { antes de la actividad? Valora de } 1 \text { a } 5 \text {, siendo uno muy escaso y } 5 \\
\text { muy completo }\end{array}$ & $\begin{array}{l}\text { Dicotómica } \\
\text { Likert }\end{array}$ \\
\hline \multirow{9}{*}{ Contenido } & $\begin{array}{l}\text { ¿Crees que la actividad Iberia refleja y permite desarrollar los } \\
\text { contenidos recogidos en el temario de la asignatura? }\end{array}$ & Dicotómica \\
\hline & $\begin{array}{l}\text { ¿Con qué elementos de la actividad crees haber desarrollado un } \\
\text { mayor aprendizaje de los contenidos históricos? }\end{array}$ & Múltiple \\
\hline & $\begin{array}{l}\text { ¿Cómo valorarías tu conocimiento sobre la Hispania prerromana } \\
\text { después de la actividad? Valora de } 1 \text { a } 5 \text {, siendo uno muy escaso } \\
\text { y } 5 \text { muy completo }\end{array}$ & Likert \\
\hline & $\begin{array}{l}\text { Con respecto a los contenidos trabajados en Iberia, señala los } \\
\text { que se han trabajado activamente }\end{array}$ & Múltiple \\
\hline & ¿Cuáles son los rasgos que más destacarías de tu personaje? & $\begin{array}{l}\text { Pregunta } \\
\text { abierta }\end{array}$ \\
\hline & ¿Has aprendido algo de él? (especifica el qué) & $\begin{array}{l}\text { Pregunta } \\
\text { abierta }\end{array}$ \\
\hline & Resume brevemente el acto I & $\begin{array}{l}\text { Pregunta } \\
\text { abierta }\end{array}$ \\
\hline & Resume brevemente el acto II & $\begin{array}{l}\text { Pregunta } \\
\text { abierta }\end{array}$ \\
\hline & Resume brevemente el acto III & $\begin{array}{l}\text { Pregunta } \\
\text { abierta }\end{array}$ \\
\hline
\end{tabular}




\begin{tabular}{|c|c|c|}
\hline \multirow{6}{*}{$\begin{array}{l}\text { Rigor } \\
\text { Histórico }\end{array}$} & $\begin{array}{l}\text { ¿Crees que todos los personajes de la actividad son personajes } \\
\text { históricos? }\end{array}$ & Dicotómica \\
\hline & $\begin{array}{l}\text { En caso de no creerlo, ¿qué porcentaje de personajes históricos } \\
\text { crees que hay? }\end{array}$ & $\begin{array}{l}\text { Pregunta } \\
\text { abierta }\end{array}$ \\
\hline & $\begin{array}{l}\text { ¿Qué facción te parece mejor representada desde el punto de } \\
\text { vista histórico? (justifica brevemente tu respuesta) }\end{array}$ & $\begin{array}{l}\text { Pregunta } \\
\text { abierta }\end{array}$ \\
\hline & ¿Has visto algún personaje femenino en juego? & Dicotómica \\
\hline & $\begin{array}{l}\text { ¿Qué grado de rigor histórico crees que tienen los personajes } \\
\text { femeninos? (Valora de } 1 \text { a } 5 \text {, siendo } 1 \text { escaso y } 5 \text { mucho) }\end{array}$ & Likert \\
\hline & $\begin{array}{l}\text { ¿Cuál crees que es el papel de la mujer en el periodo histórico } \\
\text { en que se desarrolla Iberia? }\end{array}$ & $\begin{array}{l}\text { Pregunta } \\
\text { abierta }\end{array}$ \\
\hline \multirow{4}{*}{$\begin{array}{l}\text { Percepción } \\
\text { de la } \\
\text { actividad }\end{array}$} & ¿Crees que esta actividad potencia un aprendizaje significativo? & Dicotómica \\
\hline & $\begin{array}{l}\text { De } 1 \text { a } 5 \text {, ¿cómo valorarías la experiencia en relación con } \\
\text { criterios de motivación, dinamismo y diversión en el aula } \\
\text { siendo } 1 \text { poco motivadora, poco dinámica y aburrida y } 5 \text { muy } \\
\text { motivadora dinámica y divertida? }\end{array}$ & Likert \\
\hline & $\begin{array}{l}\text { ¿Has disfrutado interpretándolo (al personaje)? (Valora de } 1 \text { a } 5 \text {, } \\
\text { siendo } 1 \text { poco o nada y } 5 \text { mucho) }\end{array}$ & Likert \\
\hline & ¿Cuál ha sido tu personaje favorito? & $\begin{array}{l}\text { Pregunta } \\
\text { abierta }\end{array}$ \\
\hline
\end{tabular}

Fuente: Elaboración propia.

En primer lugar, y tras las preguntas de control sobre procedencia y participación en el proyecto, se abordó la experiencia previa del alumnado con un ítem relacionado con la existencia de alguna experiencia previa con el LARP y otro los niveles de conocimientos previos.

La siguiente categoría que abordamos se relacionó con los contenidos aprendidos generándose un ítem que se ocupaba de los tipos de contenidos componen la actividad y otro sobre cómo se trabajan; un ítem en relación con los elementos de la actividad que ayudan al aprendizaje, otro relacionado con los contenidos desarrollados tras la actividad, dos relacionados con lo que ha aprendido del personaje y tres ítems de resumen sobre cada acto.

La siguiente categoría se centró en la valoración de la actividad en cuanto al diseño y rigor histórico, planteando dos ítems sobre: la historicidad de los personajes, uno sobre las facciones, tres sobre la representación femenina y su papel en el desarrollo histórico y otro sobre su propio personaje.

Por último, diseñamos varios ítems sobre el grado de disfrute con la actividad y la interpretación. 


\section{RESULTADOS Y DISCUSIÓN}

La actividad se llevó a cabo en el curso 2018-19 en diferentes grupos (8 de 10) tanto del centro principal como en su centro adscrito. La participación y el impacto mediático fue de gran trascendencia debido a lo novedoso del uso de la metodología docente. Como podemos ver en las fotografías el alumnado se implicó mayoritariamente por medio de la participación en la actividad y el uso de disfraces en algunos casos (Figuras 4 y 5). Se les facilitó información para que, si querían, diseñaran su propio atuendo y se les exhortó a participar en la actividad en la que el propio sistema de puntuación servía como elemento de control.

En la observación del desarrollo de la actividad se percibieron varias cuestiones de gran trascendencia:

En primer lugar, se ha de tener en cuenta que el aula como escenario para el desarrollo del LARP resultó en todos los casos un hándicap a superar debido al mobiliario fijo y la falta de adecuación a una actividad en la que grupos de 20 a 50 personas debían desplazarse y conversar en un espacio cerrado.

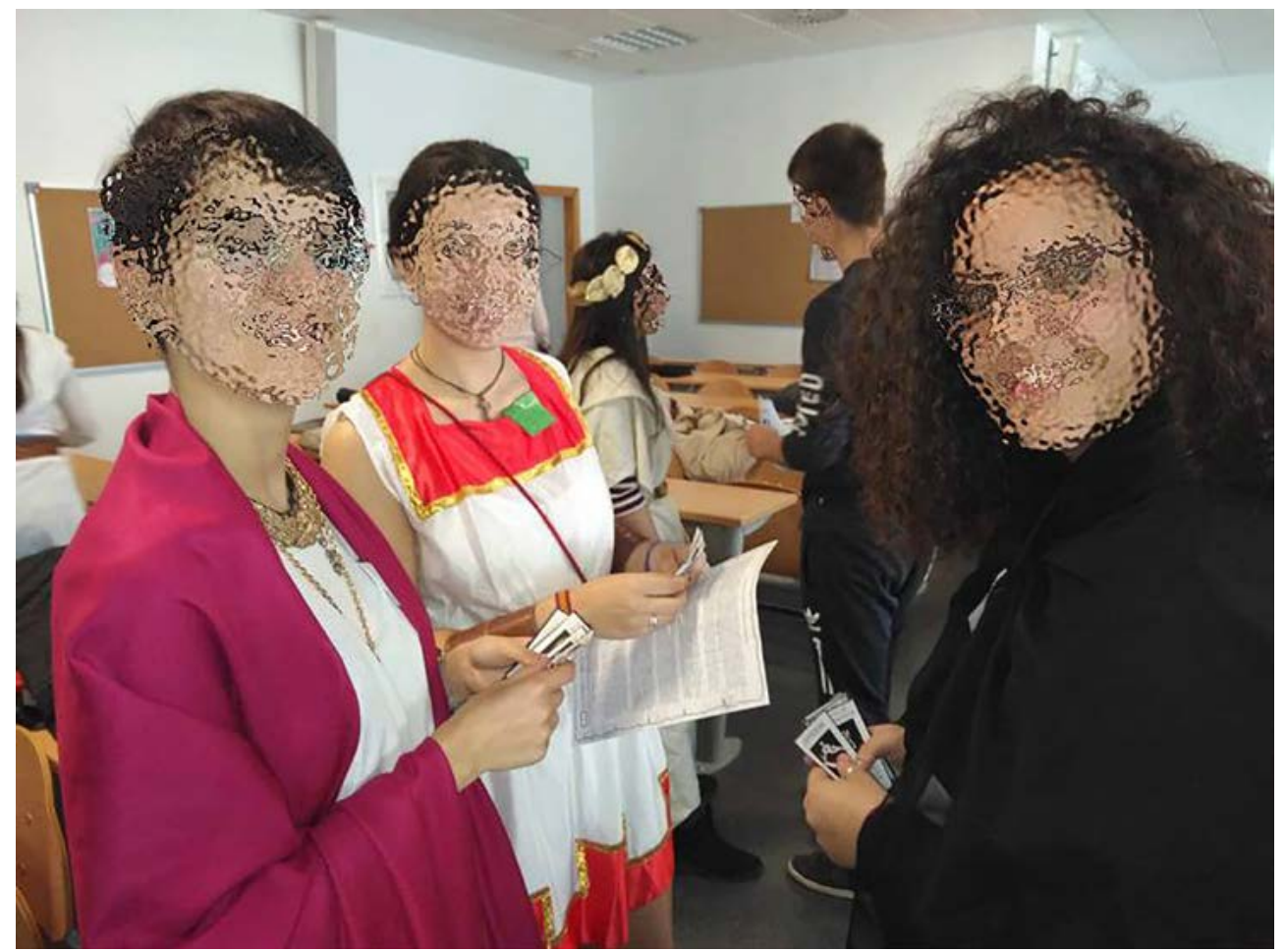

Figura 4. Fotografía alumnos disfrazados. 


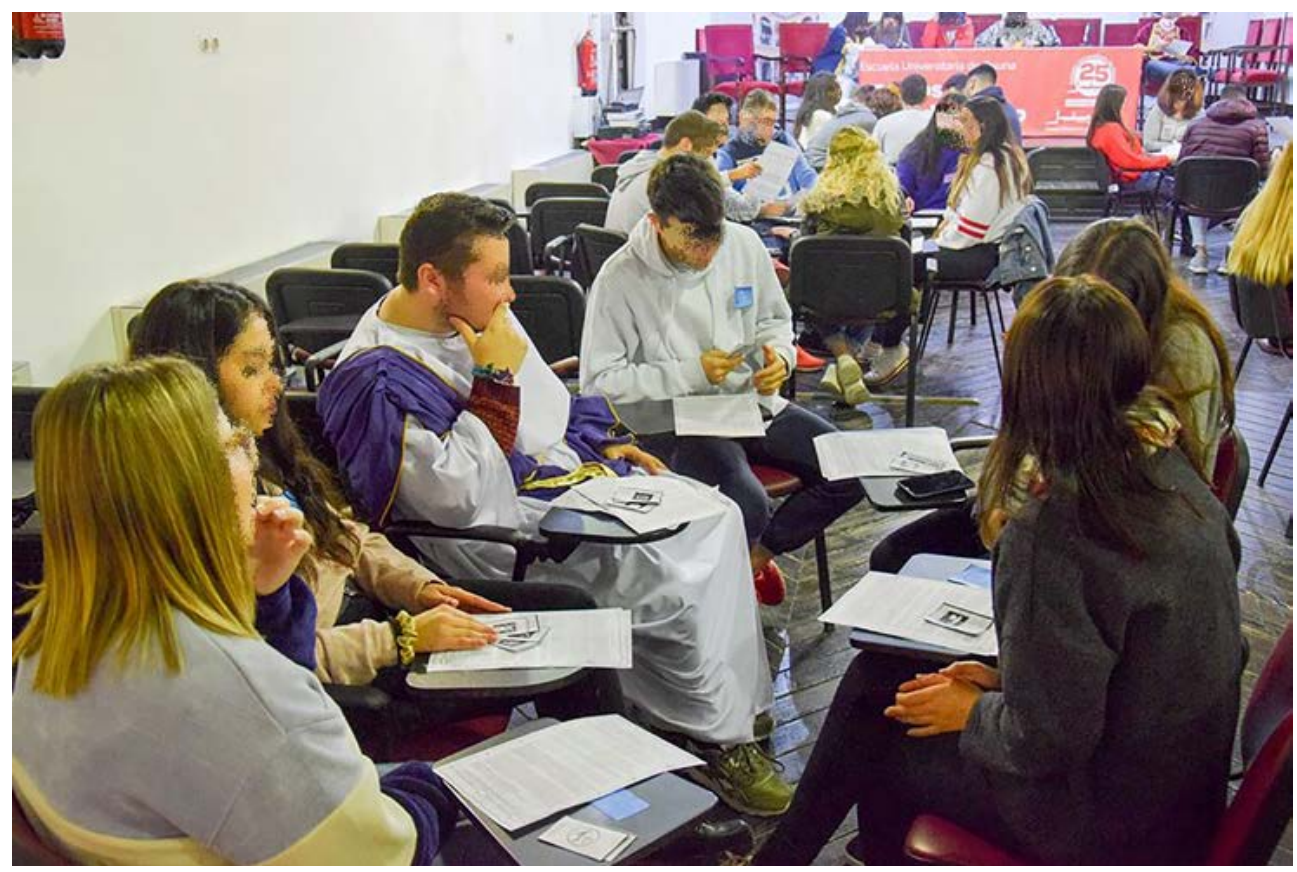

Figura 5. Fotografía alumnos disfrazados.

En segundo lugar, relacionado también con la problemática planteada por el entorno en el que se desarrollaba el juego, hemos de resaltar que el uso de parlamentos para mantener la narrativa e informar a los jugadores de hechos históricos se vio altamente afectado, ya que muchos alumnos no pudieron atender con atención a los monólogos que se expusieron.

En tercer lugar, en relación con el sistema de juego, debemos decir que resultó muy llamativo para el alumnado. El sistema de intercambio de cartas para recolectar recursos fue un elemento altamente dinamizador de la actividad, así como las cartas de intervención repartidas a partir del acto II. Sin embargo, la sencillez de la mecánica terminó por empujar al alumnado a centrarse en la actividad del cambio de cartas en vez de analizarlas y comprender para qué servían o si guardaban relación con las narrativas de los personajes que interpretaban.

Por último, el profesorado se sorprendió del aumento del interés y la participación por parte del alumnado conforme pasaron las tres sesiones, siendo especialmente sorprendente el estupor y la sorpresa de algunos alumnos al conocer el desenlace de los acontecimientos históricos, demostrando el escaso conocimiento que se tenía de un periodo estudiado en la educación obligatoria y que debían recordar.

Además, de la observación en el sistema de prácticas se tomaron tres grupos para realizar un cuestionario diseñado exprofeso para indagar sobre las percepciones de los participantes. De los grupos participantes no todos los alumnos 


\section{¿Has participado alguna vez en una actividad de LARP (rol en vivo) o rol play masiva?}

92 respuestas

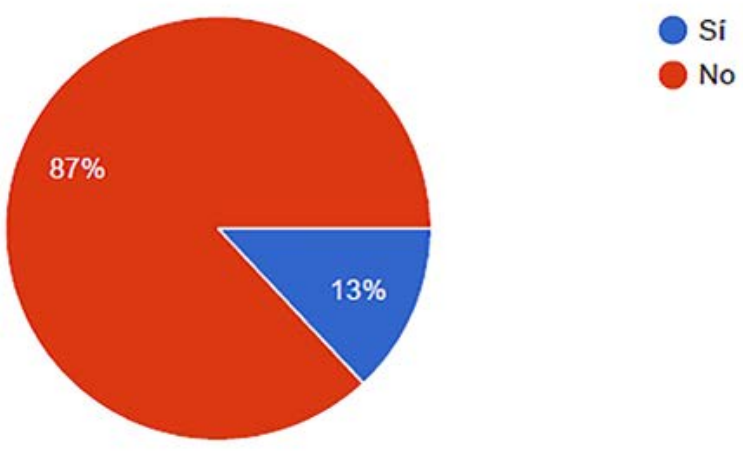

Figura 6. Grafica de experiencia en LARPS. Elaboración propia.

rellenaron el formulario, pero se obtuvo una muestra representativa de 92 encuestados que presentaron respuestas de las que destacamos:

En primer lugar, solo un $13 \%$ de los encuestados había realizado alguna actividad de LARP previamente, lo que confirma la problemática y necesidad de adaptación de nuestro recurso para principiantes (Figura 6).

En segundo lugar, en relación con la valoración de la actividad se analizaron las percepciones del alumnado desde diferentes puntos de vista. Así, en lo referente a la motivación, solo un $18,4 \%$ valoró la actividad como poco motivadora y poco divertida, frente al $53,2 \%$ que la valoró bastante o muy motivadora y divertida y un $28,3 \%$ que mostró una opinión intermedia (Figura 7). Con respecto al grado de disfrute en la interpretación de sus personajes, un 45,1\% disfrutaron mucho o bastante de la actividad, un $29,7 \%$ contestó con una respuesta de grado intermedio y solo un $25 \%$ disfrutó poco o nada con la interpretación de su personaje (Figura 8). Además, en cuanto a la valoración del aprendizaje a nivel de contenidos, y frente a las preguntas de si la actividad reflejaba y permitía desarrollar los contenidos recogidos en el temario de la asignatura y si potenciaba un aprendizaje significativo, el alumnado contestó positivamente a la primera en una relación porcentual de $67,4 \%$ a favor del desarrollo de contenidos propios del temario y un $76,1 \%$ a favor de que la actividad genera un aprendizaje significativo.

En tercer lugar, en relación con los contenidos teóricos trabajados, debemos resaltar que un $73,6 \%$ ( $45,1 \%$ valoran 1 y $28,6 \%$ valoran en 2 sobre 5) afirmaba tener un conocimiento escaso o nulo de los contenidos que se abordaban en la actividad antes de realizarla (Figura 9) y que tras la misma se aprecia una notable mejora al descender a más de la mitad ese porcentaje $(30,4 \%$ de los que $8,7 \%$ valoran en 1 sobre 5 y $21,7 \%$ en 2 sobre 5 . Figura 10). 
De 1 a 5, ¿cómo valorarias la experiencia en relación con criterios de motivación, dinamismo y diversión en el aula siendo 1 poco motivadora, poco dinámica y aburrida y 5 muy motivadora dinámica y divertida? 92 respuestas

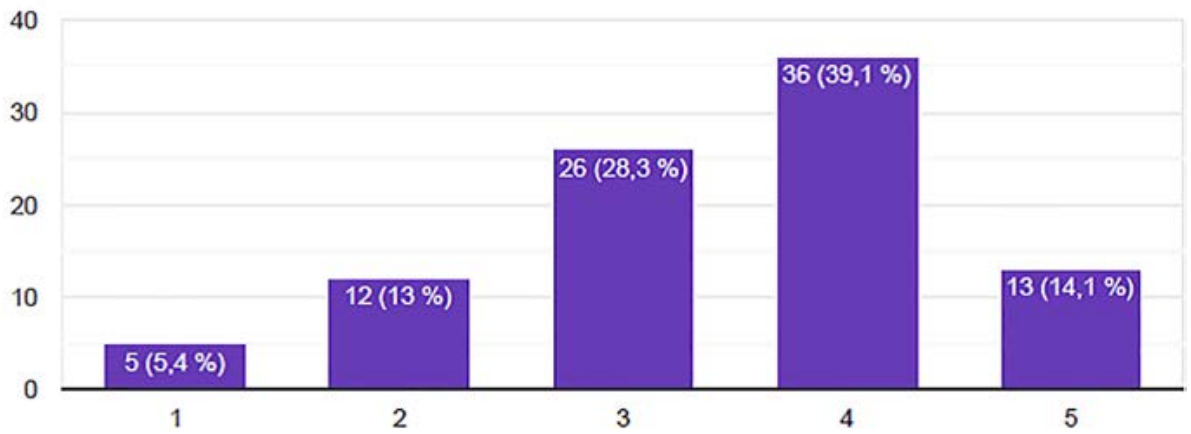

Figura 7. Gráfica de motivación. Elaboración propia.

¿Has disfrutado interpretándolo? (Valora de 1 a 5 , siendo 1 poco o nada y 5 mucho) 91 respuestas

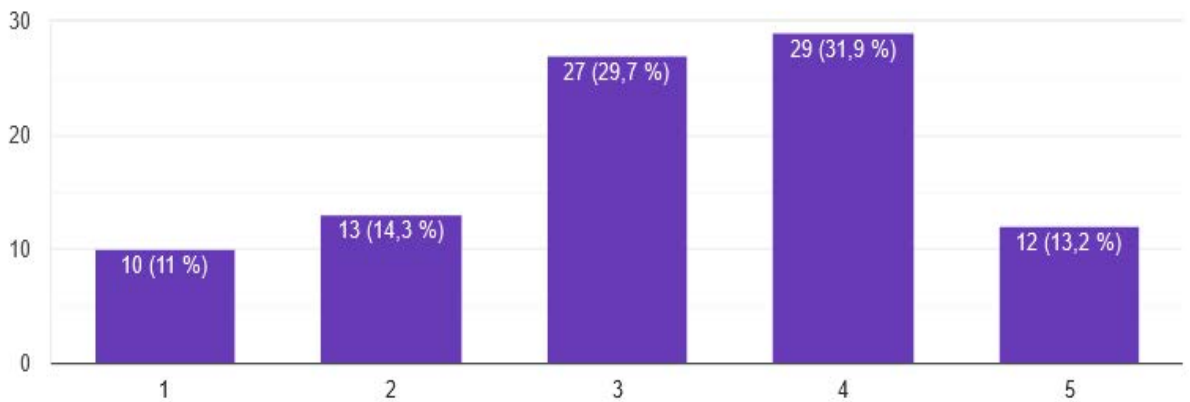

Figura 8. Gráfica Disfrute del personaje. Elaboración propia.

Además, como hemos mencionado, un $67 \%$ entiende que la actividad les ha permitido desarrollar los contenidos del temario de la asignatura, resaltando en otra de las preguntas los elementos políticos y económicos (Figura 11). 
¿Cómo valorarias tu conocimiento sobre la Hispania prerromana antes de la actividad? Valora de 1 a 5 siendo uno muy escaso y 5 muy completo 91 respuestas

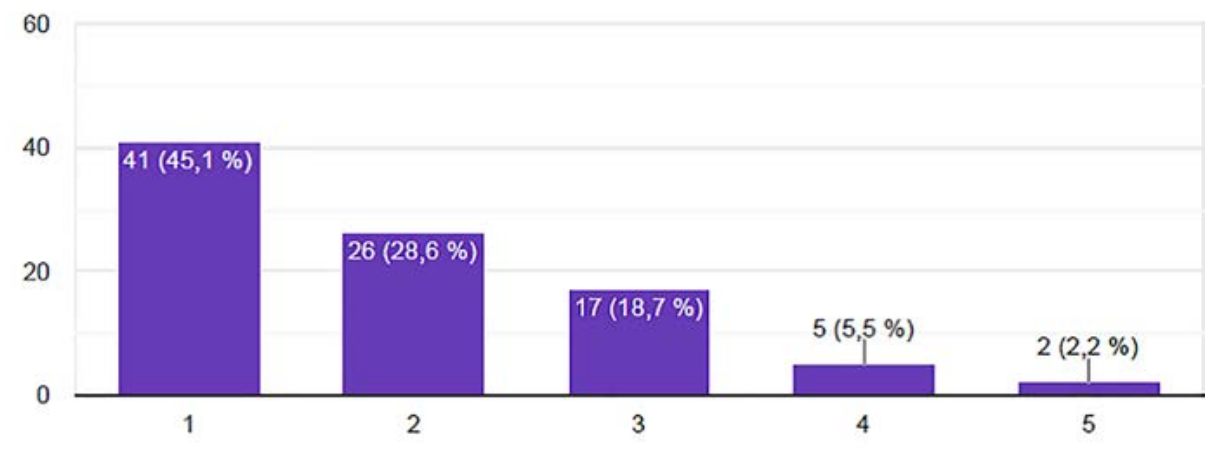

Figura 9. Grafica de conocimientos previos. Elaboración propia.

¿Cómo valorarias tu conocimiento sobre la hispania prerromana despues de la actividad? Valora de 1 a 5 siendo uno muy escaso y 5 muy completo 92 respuestas

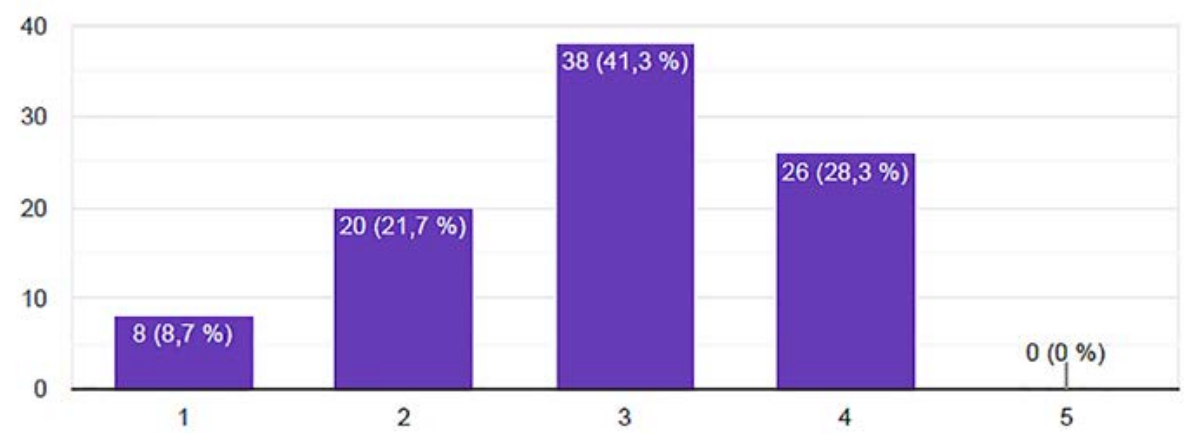

Figura 10. Grafica de contenidos adquiridos. Elaboración propia. 
Con respecto a los contenidos trabajados en Iberia, señala los que se han trabajado activamente

90 respuestas

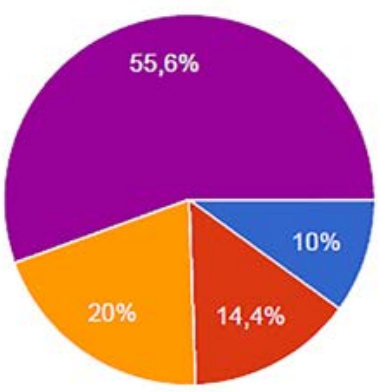

Contenidos sobre la

organización social

Contenidos sobre la

organización politica

Contenidos sobre la economia de los pueblos de Iberia

Contenidos sobre las diferentes religiones

Contenidos sobre los hechos politicos más relevantes (bata..

Figura 11. Grafica de contenidos aprendidos. Elaboración propia.

En cuarto lugar, con relación a las preguntas abiertas, hemos detectado una cierta reticencia a la hora de responderlas. En muchas de ellas el número de respuestas recibidas es de un $60-70 \%$ del total, porcentaje que se reduce cuando se solicita los resúmenes de cada sesión. Además, un número elevado de las respuestas recibidas han sido contestadas obviando las instrucciones, reduciendo así la información que aportan. No obstante, se aprecia un elevado interés del alumnado por la actividad: se aprecia que, pese a lo observado en clase, han valorado positivamente las narrativas desarrolladas a través de los parlamentos, aunque critican la polarización del protagonismo, que recae principalmente en las facciones romana y cartaginesa, siendo el mejor reflejo que los personajes más valorados hayan sido Aníbal, Asdrúbal y Escipión.

Por último y como sistema de evaluación final, se incorporaron al examen las preguntas propias de este proyecto, así como de otro proyecto de diseño de materiales lúdicos centrados en la Antigüedad Tardía. En este ejercicio no se apreció un cambio significativo en las evaluaciones con respecto al curso previo, manteniéndose los porcentajes de aprobados y suspensos. Entendemos que esta situación se pudo deber a dos factores: en primer lugar, el uso de un sistema tradicional de preguntas de desarrollo no adaptado a las prácticas, y segundo, la posible creencia del alumnado en que al haberse trabajado el temario en las clases prácticas preguntas de estos temas no entrarían en el examen. 


\section{REFLEXIONES FINALES}

Tras el análisis de los resultados, y a modo de conclusión, quisiéramos valorar de forma muy positiva el diseño y aplicación del recurso didáctico Iberia. Así pues, y teniendo en cuenta la novedad del sistema del Edu-LARP, el cual nunca había sido puesto en práctica por el profesorado de la asignatura, así como la falta de preparación previa debido a los plazos propios de los proyectos de innovación, podemos valorar el grado de participación del profesorado y alumnado, así como el proceso de aplicación como muy satisfactorio. El alumnado se ha mostrado muy participativo y ha presentado un aumento de interés por la asignatura. Se podría decir que se ha conseguido crear una actividad inmersiva en la que se han trabajado contenidos propios de la asignatura de manera directa, activa y participativa. De manera general el alumnado ha definido su proceso de aprendizaje como «significativo» y si bien estos resultados han tenido poco impacto en la evaluación final, entendemos que no por ello debemos tomar esta actividad como un fracaso.

Al contrario de lo que pudiera plantearse, nuestro objetivo tras este proyecto es depurar la actividad en los aspectos que más deficiencias haya presentado, como el sistema de evaluación y la trasposición de los contenidos prácticos a un modelo de examen aún centrado en el aprendizaje memorístico.

Entendemos que es necesario potenciar los procesos de reflexión y análisis con respecto a la actividad para potenciar el desarrollo de aprendizaje incorporando sistemas de informes o diarios de prácticas, instrumentos usados muy a menudo por este alumnado en las asignaturas como Prácticum. Otro elemento que revisar será el sistema de juego, que debemos repasar y vincular de manera directa con las narrativas de cada personaje, de forma que el participante deba profundizar algo más en las mismas para entender la evolución del juego.

Así mismo, en relación con los instrumentos utilizados, entendemos que sería necesario su perfeccionamiento en aspectos de validación del cuestionario y significatividad de la muestra. Google Forms es una herramienta que tiene un gran potencial, sin embargo, el desarrollo de los cuestionarios online tiene, desde nuestra perspectiva, la problemática de la voluntariedad por parte del participante, que, si bien debemos respetar, en los formatos online presentan una problemática doble, tanto técnica, frente a la comprensión y respuesta de las preguntas, como temporal, entendiendo la falta de tiempo dedicado a la participación fuera del horario lectivo.

Todas estas consideraciones se tendrán en cuenta para futuros proyectos, los cuales no han podido plantearse en los dos últimos cursos debido a la problemática derivada de la situación sanitaria actual. Así, no descartamos ampliar el uso de Iberia a otras titulaciones e incluso abrirlo al uso en otros centros universitarios o en su defecto generar nuevos recursos de Edu-LARP que permitan acceder al profesorado universitario a una herramienta didáctica, fácil, sencilla que solvente los problemas de motivación y participación en las prácticas de las asignaturas de Historia e Historia Antigua. 


\section{REFERENCIAS BIBLIOGRÁFICAS}

Alarcón Hernández, C. (2016). Claves para la enseñanza de los Fundamentos de Historia de España en el Grado de Educación Primaria. En R. Porlán y E. Navarro (coords.), Libro de Actas III Jornadas de Docencia Universitaria (pp. 886-895). Madrid: Ediciones Morata.

Alfaro Rocher, I. J. (2005). El Espacio Universitario Europeo: Entre la autonomía, la diversidad y la convergencia. Cuadernos de Integración Europea, 2, pp. 3-15. http://repositori.uji.es/xmlui/bitstream/handle/10234/120189/espacio_ alfaro_2005.pdf?sequence=1

Altamirano Martínez J. V. (2016). ¿Esto es un juego? juegos serios y gamificación. Taxonomía y aplicación. Trabajo de Fin de Máster, Universidad Politécnica de Valencia.

Álvarez, Melero, A., Álvarez-Ossorio, Rivas, A., Cidoncha, Redondo, F., y Sánchez Domínguez, V. (2018). El uso del Kahoot y del Jumble como herramienta de trabajo para la enseñanza para la Historia Antigua y Medieval de España. En M. Rodríguez López y R. Anguita Martínez, (eds.), Innovaciones en el aprendizaje con tecnologías digitales, (pp. 97-108). Camas: Egregius.

Amézquita-Castañeda, I., Moreno-Ramos, M. T. (2001). La odisea liberadora de los juegos de rol. Tlaquepaque, Jalisco. México: ITESO, Consejo Nacional para la Enseñanza y la Investigación de las Ciencias de la Comunicación, A. C. (CONEICC).

Benito, Á. y Cruz, A. (coords.). (2012). Nuevas claves para la Docencia Universitaria en el Espacio Europeo de Educación Superior. Madrid: Narcea.

Bowman, S. L., (2010), The functions of role-playing games: How participants create community, solve problems, and explore identity. Jefferson, NC: McFarland and Company, Inc.

Bowman, S. L., y Standiford, A. (2015). Educational larp in the middle school classroom: A mixed method case study. International Journal of Role-Playing, 5(1), pp. 4-25. http://ijrp.subcultures.nl/wp-content/uploads/2016/12/IJRP-5Bowman-and-Standiford.pdf 
Calvo, A. y Mingorance, A. C. (2009). La estrategia de las universidades frente al Espacio Europeo de Educación Superior. Revista Complutense de Educación, 20(2), pp. 319-34. https://dx.doi.org/10.5209/RCED

Carbó García, J. R., y Pérez Miranda, I. (2010). Fuentes históricas de los juegos de rol: un experimento para la didáctica de la historia antigua. Education in the Knowledge Society (EKS), 11(3), pp. 149-167. https://doi.org/10.14201/ eks.7454

Cárdenas, J. F. (2015). La armonización en el Espacio Europeo de Educación Superior: El estado de la cuestión. Journal of Supranational Policies of Education, 3, pp. 264-282. http://hdl.handle.net/10486/667468

Cardete del Olmo, M. C. (2011). El uso del Campus Virtual para la enseñanza de la historia antigua a través del aprendizaje cooperativo. En A. Sanz Cabrerizo, J. A., López Orozco y L. Pablo Núñez (eds.), VI Jornada Campus Virtual UCM: Campus Virtual crece: retos del EEES y oportunidades para la UCM. Universidad Complutense de Madrid (pp. 143-149). Madrid: Editorial Complutense de Madrid.

Craig A., L., y Eladhari, M. (2005). Narrative structure in trans-reality role playing games: integrating story construction from live action, table top and computerbased role-playing games. Gotland University. DiGRA 2005, Cramergatan.

De Miguel, M. (2006). Metodologías de enseñanza y aprendizaje para el desarrollo de competencias. Orientaciones para el profesorado universitario ante el espacio europeo de educación superior. Madrid: Alianza.

De-Alba-Fernández, N. y Porlán, R. (2017). La metodología de enseñanza. En R. Porlán (coord.), Enseñanza universitaria. Cómo mejorarla (pp. 37-54). Madrid: Editorial Morata.

De-Alba-Fernández, N., Navarro-Medina, E., Porlán, R. y Rodríguez-Pérez, N. (2020). La progresión de los modelos docentes universitarios: resultados del primer estudio. En N. De-Alba-Fernández, y R. Porlán (coords.), Docentes universitarios. Una formación centrada en la práctica (pp. 191-230). Madrid: Editorial Morata.

Dewey, J. (1997). How we think, New York. 
Espinel Souares, A. (2007). Problemas de enseñanza y aprendizaje de historia universal I (sociedades antiguas). Revista Docencia Universitaria, 8(1), pp. 139-154.

Gómez Carrasco, C. J., Ortuño Molina, J. y Molina Puche, S. (2014) Aprender a pensar históricamente. Retos para la historia en el siglo XXI Revista Tempo e Argumento, vol. 6. 11. 5-27. https://www.redalyc.org/articulo. oa? id=338131531002

Gómez F. (2018 A). Innovación en la didáctica de la Historia: Del manual al libro 2.0. Nuevos modelos de divulgación para nuevas necesidades educativas. En O. Buzón García (ed.), Innovación y tendencias educativas: un camino hacia las nuevas formas de aprendizaje (pp. 161-187). Camas: Egregius.

Gómez-Carrasco, C. J. G., y Miralles-Martínez D, P. (2015) ¿Pensar históricamente o memorizar el pasado? La evaluación de los contenidos históricos en la educación obligatoria, en España. Revista de estudios sociales, (52), pp. 52-68. https://doi.org/10.7440/res52.2015.04.

Huizinga, J. (2000). Homo ludens. Madrid:Alianza.

Jiménez Hernández, D., González Ortiz, J.J. y Tornel Abellán, M. (2020). Metodologías activas en la universidad y su relación con los enfoques de enseñanza. Profesorado. Revista de Currículum y Formación de Profesorado, 24(1), pp. 76-94. https://doi.org/10.30827/profesorado. v24i1.8173

Lee, J. J., y Hammer, J. (2011). Gamification in education: What, how, why bother? Academic Exchange Quarterly, 15(2), 146-151.

Mackay, D. (2001). The Fantasy Role-Playing Game: A New Performing Art. Jefferson. Carolina del Norte.

Maragliano, A. (2019). Edu-larp Paths in Education: A Pedagogic Research on Ethnic Prejudice and Empathy through Games. En 9th International Conference the Future of Education. McFarland \& Company, Inc. https:// conference.pixel-online.net/FOE/files/foe/ed0009/FP/5814-LGM3956FP-FOE9.pdf 
Mochocki, M. (2013). Edu-larp as revision of subject-matter knowledge. International Journal of Role-Playing, 4, pp. 55-75. http://www.ijrp. subcultures.nl/wp-content/issue4/IJRPissue4mochocki.pdf

Prieto Navarro, L., Blanco, A., Morales Vallejo, P., \& Torre Puente, J. C. (2008). La enseñanza universitaria centrada en el aprendizaje. Estrategias para el profesorado. Barcelona: Octaedro-Universitat de Barcelona. Institut de Ciències de l'Educació.

Qian, M., y Clark, K. R. (2016). Game-based Learning and $21^{\text {st }}$ century skills: A review of recent research. Computers in Human Behavior, 63, pp. 50-58. https://doi.org/10.1016/j.chb.2016.05.023

Rangel Jiménez, M. (2015). Los juegos de rol, herramienta comunicativa generadora de narrativas hiperreales interactivas. (Avance de investigación, estado del arte). En Solís E. Hernández, y R. Domínguez Cortina (coords.), Historias y aportes sociales de la investigación de la comunicación en México: ¿Cuáles son los acuerdos mínimos del núcleo disciplinario? Memorias XXVII Encentro Nacional de la AMIC, Querétaro. http://amic2015.uaq.mx/docs/memorias/GI_11_PDF/GI_11_Los_ juegos-_de_rol.pdf

Roda, A. (2010). Juego de rol y educación, hacia una taxonomía general, Education in the Knowledge Society (EKS), 11(3), pp. 185-204. https:// doi.org/10.14201/eks.7458

Ruiz López I. D. (2020). La imagen de la Historia Antigua en los futuros docentes de Educación Primaria. Revista UNES. Universidad, Escuela y Sociedad, (9), pp. 74-88. https://doi.org/10.30827/unes.v0i9.15966

San Bernardino Coronil, J. (2019). La enseñanza y aprendizaje en Historia Antigua de Asia Oriental. En R. Porlán Ariza y E. Navarro-Medina (coords.), Construyendo nuevas estrategias de innovación docente (pp. 25722598). Sevilla: Secretariado de Formación y Evaluación de la Universidad de Sevilla. https://doi.org/10.12795/9788447221912.116

Sánchez Domínguez V. (2018). Roma un juego de rol para vivenciar la vida en la República y en el Imperio Romano. En A. Torres-Toukoumidis y L. M. Romero Rodríguez (coords.), Gamificación en Iberoamérica: Experiencias desde la educación y la comunicación (pp. 349-375). Quito: Abya Yala. 
Sánchez Domínguez V. (2019.) Los juegos de rol y la gamificación un viaje de ida y vuelta. En D. Moya López, (coord.), Arterias de la sociedad del siglo XXI. las TIC en el mundo como herramienta multidisciplinar: estudios de caso (pp. 197-220). Camas: Egregius.

Sánchez Domínguez, V., Álvarez-Ossorio Rivas, A. y Lozano Gómez, F. (2019). Nuevos alumnos, nuevos retos y recursos diferentes: un libro digital para la enseñanza de la Historia Antigua. El Futuro del Pasado. Revista electrónica de Historia, 10, pp. 343-372. https://doi.org/10.14516/ fdp.2019.010.001.013

Sánchez Domínguez, V., Alvarez-Ossorio Rivas, A., Alarcón Hernández, C. y Lozano Gómez, F. (2017). Innovación en la didáctica de la Historia: Del manual al libro 2.0. Nuevos modelos de divulgación para nuevas necesidades educativas. En O. Buzón García (ed.), Innovación y Tendencias Educativas:un camino hacia las nuevas formas de aprendizaje (pp. 161186). Sevilla: Egregius.

Torstein, U. (2005). Live Action Role Playing, Teaching through gaming. Dissecting Larp, pp. 23-30. http://citeseerx.ist.psu.edu/viewdoc/downl oad?doi=10.1.1.591.1171\&rep=rep1\&type=pdf

Vanek, A., y Peterson, A. (2016). Live action role-playing (LARP): Insight into an underutilized educational tool. En K. Schrier (ed.), Learning, Education and Games, Volume Two: Bringing Games into Educational Contexts (pp. 219-240). ETC Press.

Vázquez García, Juan A. (2015). Nuevos escenarios y tendencias universitarias. Revista de Investigación Educativa, 33(1), 13-26. https:// doi.org/10.6018/rie.33.1.211501

Vygotsky, L. S. (1982). El juego y su función en el desarrollo psíquico del niño. Revista Cuadernos de Pedagogía, 85, pp. 39-49. 
